\title{
ON INFINITE HORIZON MULTI-PERSON STOPPING GAMES WITH PRIORITIES
}

\author{
ELŻBIETA Z. FERENSTEIN \\ Faculty of Mathematics and Information Science, Warsaw University of Technology \\ Plac Politechniki 1, 00-661 Warszawa, Poland \\ E-mail: efer@mini.pw.edu.pl
}

\begin{abstract}
We study nonzero-sum multi-person multiple stopping games with players' priorities. The existence of Nash equilibrium is proved. Examples of multi stopping of Markov chains are considered. The game may also be presented as a special case of a stochastic game which leads to many variations of it, in which stopping is a part of players' strategies.
\end{abstract}

1. Introduction and preliminaries. The paper is concerned with a $k$-person, $k \geq 2$, nonzero-sum noncooperative $k$ stage sequential game in which players strategies consist of $k$ stopping times. Players' indices $1,2, \ldots, k$ correspond to their ordering (ranking) called priorities so that 1 refers to the player with the highest priority and $k$ to the lowest one. At the first stage of the game only one reward from the sequence of rewards $\left\{X_{n}^{1}, n \in N\right\}$ may be selected by one of the players in a sequential way like in the best choice problems (secretary type problems), that is at any time $n$ if a reward has not been selected by that time Player 1 makes a decision: to select the reward $X_{n}^{1}$ or to reject it. In the former case, stage 1 of the game is over. Player 1 quits the game with the selected reward and there are $k-1$ remaining players observing step by step elements of the new sequence of rewards $\left\{X_{n+j}^{2}, j \in N\right\}$. On the other hand if Player 1 has rejected the reward then Player 2 has the same options as Player 1. If he rejects the reward Player 3 exercises the same options as his predecessors and so on until there is a player who decides to take the reward. Equivalently one may say that the reward $X_{n}^{1}$ is selected by the player with the highest priority among those who decide to stop the first stage of the game at time $n$ and to take it. If all players reject it the same selection scheme is applied at time $n+1$ when the reward $X_{n+1}^{1}$ is presented for selection. The first stage of the game is finished as soon as one of the players selects a reward at some random time $t_{1}$, say. Suppose $t_{1}=n_{1}$. At stage 2 , in a similar sequential way, one reward from

2000 Mathematics Subject Classification: Primary 60G40.

The paper is in final form and no version of it will be published elsewhere. 
the sequence $\left\{X_{n_{1}+j}^{2}, j \in N\right\}$ is selected by one of the remaining $k-1$ players with the highest priority among those who have decided to take the reward $X_{t_{2}}^{2}$, at some random time $t_{2}$, that is, rewards $X_{n_{1}+1}^{2}, X_{n_{1}+2}^{2}, \ldots, X_{t_{2}-1}^{2}$ have been rejected. In the final stage $k$ of the game there is one player left and supposing that $t_{k-1}$ is the time of stopping the previous stage of the game, he faces the problem of selecting the stopping time $t_{k} \geq$ $t_{k-1}+1$, a.s., resulting in getting the reward $X_{t_{k}}^{k}$. Each player aims to get his mean reward as large as possible. So, we consider the problem of finding Nash equilibrium strategies in the class of admissible sequential strategies described above in slightly informal way. A precise model of the game will be presented in Section 2.

The described game captures the following trading situation. The owner has $k$ commodities to sell. The prices of commodities are random and vary over time. Let $X_{n}^{j}$ denote the price of the $j$-th commodity at time $n, n=1,2, \ldots$ The owner may sell commodities in a subsequent way as follows. First, commodity 1 may be sold, at any random time $t_{1}$, say. Then, at a random time $t_{2}>t_{1}$, a.s., commodity 2 may be sold, and so on, until at some random time $t_{k}, t_{k}>t_{k-1}>\cdots>t_{1}$, a.s., commodity $k$ is sold. Random times of buying commodities $t_{1}, \ldots, t_{k}$ are chosen by $k$ ordered buyers, in the way described above. The aim of each buyer is to purchase a commodity at the lowest price. Another possible applications of the game are in queueing or telecommunication systems, job allocations.

Many variations of stopping games have been investigated in the literature since the paper by Dynkin (1969).

These games are generalizations of the stopping game formulated by Dynkin as an example of optimal stopping of random sequences. First we will quote main results on stopping games with players strategies belonging to a class of (nonrandomized) stopping times.

Let $(\Omega, \mathcal{F}, P)$ be an underlying probability space on which all considered random variables are defined and $\left\{\mathcal{F}_{n}, n \in N\right\}$ an increasing sequence of sub- $\sigma$-fields of $\mathcal{F}, N=$ $\{1,2, \ldots\} . \mathcal{F}_{n}$ is a $\sigma$-field of events observed by the players till time $n, n \in N$, inclusively. Let $M$ denote the set of stopping times (Markov times) $\tau$ with respect to $\left\{\mathcal{F}_{n}, n \in \bar{N}\right\}$, $\bar{N}=N \cup\{\infty\}, \mathcal{F}_{\infty}=\sigma\left(\left\{\mathcal{F}_{n}, n \in N\right\}\right.$, that is $\tau: \Omega \rightarrow \bar{N}$ and $\{\omega \in \Omega: \tau(\omega)=n\} \in \mathcal{F}_{n}$, for any $n \in \bar{N}$.

Dynkin considered the following zero-sum stopping game. Two players observe an $\left\{\mathcal{F}_{n}, n \in N\right\}$ adapted bivariate sequence $\left\{\left(X_{n}, \varphi_{n}\right), n \in N\right\}$ of random variables. Player 1 and Player 2 have the strategy sets $M \cap\left\{\tau: \varphi_{\tau} \leq 0\right\}$ and $M \cap\left\{\tau: \varphi_{\tau}>0\right\}$, respectively. Let $\left(\tau_{1}, \tau_{2}\right)$ be a pair of players' strategies. The game terminates at $\tau=\min \left\{\tau_{1}, \tau_{2}\right\}$ and $X_{\tau}$ is the reward (loss) for Player 1 (Player 2). The objective of Player 1 (Player 2) is to maximize (minimize) the mean $E\left(X_{\tau}\right)$. Neveu (1975) considered a slight modification of this zero-sum stopping game in which the game strategy is a pair of stopping times $\left(\tau_{1}, \tau_{2}\right) \in M \times M$ and the reward (loss) $R\left(\tau_{1}, \tau_{2}\right)$ for Player 1 (Player 2) is determined by an $\left\{\mathcal{F}_{n}, n \in N\right\}$ adapted bivariate sequence $\left\{\left(X_{n}, Y_{n}\right), n \in N\right\}$ of random variables so that

$$
R\left(\tau_{1}, \tau_{2}\right)=X_{\tau_{1}} I_{\left\{\tau_{1}<\tau_{2}\right\}}+Y_{\tau_{2}} I_{\left\{\tau_{2} \leq \tau_{1}\right\}},
$$

where $I_{A}$ denotes the indicator function of the set $A$ in $\mathcal{F}$. The game payoff is $V\left(\tau_{1}, \tau_{2}\right)=$ 
$E\left(R\left(\tau_{1}, \tau_{2}\right)\right)$. The game has a value and max-min strategies under suitable integrability assumptions and $X_{n} \leq Y_{n}$, a.s., $n \in N$. Zero-sum stopping games with more general than (1.1) forms of the reward were investigated by Yasuda (1985) and Rosenberg, Solan and Vieille (2000). Let $\left\{\left(X_{n}, Y_{n}, W_{n}\right), n \in N\right\}$ be an $\left\{\mathcal{F}_{n}, n \in N\right\}$ adapted sequence of the observed reward related random variables satisfying the following condition

$$
E\left(\sup _{n \in N} \max \left\{\left|X_{n}\right|,\left|Y_{n}\right|,\left|W_{n}\right|\right\}\right)<\infty .
$$

Let the reward (loss) for Player 1 (Player 2) under the strategy $\left(\tau_{1}, \tau_{2}\right)$ be as follows

$$
R\left(\tau_{1}, \tau_{2}\right)=X_{\tau_{1}} I_{\left\{\tau_{1}<\tau_{2}\right\}}+Y_{\tau_{2}} I_{\left\{\tau_{2}<\tau_{1}\right\}}+W_{\tau_{1}} I_{\left\{\tau_{1}=\tau_{2}\right\}},
$$

and the discounted reward with $\lambda \in(0,1)$ :

$$
R_{\lambda}\left(\tau_{1}, \tau_{2}\right)=\lambda^{\tau_{1}} X_{\tau_{1}} I_{\left\{\tau_{1}<\tau_{2}\right\}}+\lambda^{\tau_{2}} Y_{\tau_{2}} I_{\left\{\tau_{2}<\tau_{1}\right\}}+\lambda^{\tau_{1}} W_{\tau_{1}} I_{\left\{\tau_{1}=\tau_{2}\right\}} \cdot
$$

Unless the inequalities $X_{n} \leq W_{n} \leq Y_{n}$, a.s., $n \in N$, are fulfilled these games may not have values. It turns out that without this assumption one may get existence of a game value in the class of randomized stopping times. Existence of a value of a zero-sum game with randomized stopping times and discounted reward $R_{\lambda}\left(\tau_{1}, \tau_{2}\right)$ was proved by Yasuda (1985). Rosenberg, Solan and Vieille (2000) showed that in the class of randomized stopping times the game with a reward (1.3) has a value. Moreover, they showed that a game value $V\left(\tau_{1}, \tau_{2}\right)$ is the limit of game values $V_{\lambda}\left(\tau_{1}, \tau_{2}\right)$ as $\lambda \rightarrow 0^{+}$of games with discounted rewards $R_{\lambda}\left(\tau_{1}, \tau_{2}\right)$.

Nonzero-sum stopping games with general rewards (1.5), given below, satisfying the integrability condition (1.2) were investigated by Ohtsubo (1987, 1991). Let the reward for Player $i, i=1,2$, under the game strategy $\left(\tau_{1}, \tau_{2}\right) \in M \times M$ be as follows

$$
R^{i}\left(\tau_{1}, \tau_{2}\right)=X_{\tau_{i}}^{i} I_{\left\{\tau_{i}<\tau_{j}\right\}}+Y_{\tau_{j}}^{i} I_{\left\{\tau_{j}<\tau_{i}\right\}}+W_{\tau_{i}}^{i} I_{\left\{\tau_{i}=\tau_{j}<\infty\right\}}+\limsup _{n \rightarrow \infty} W_{n}^{i} I_{\left\{\tau_{1}=\tau_{2}=\infty\right\}},
$$

where sequences of trivariate random variables $\left\{\left(X_{n}^{i}, Y_{n}^{i}, W_{n}^{i}\right), n \in N\right\}$ are $\left\{\mathcal{F}_{n}, n \in N\right\}$ adapted. They represent players' rewards associated with their appropriate decisions. The aim of each of the players is to make his mean reward as large as possible. So, they look for a Nash equilibrium strategy $\left(\widehat{\tau}_{1}, \widehat{\tau}_{2}\right) \in M \times M$ of the game $\mathcal{G}=\left(M \times M, V^{1}, V^{2}\right)$, presented in a normal form, where players' payoff functions $V^{i}$ are their mean rewards, that is $V^{i}\left(\tau_{1}, \tau_{2}\right)=E\left(R^{i}\left(\tau_{1}, \tau_{2}\right)\right), i=1,2$. Thus, for any strategy $\left(\tau_{1}, \tau_{2}\right)$ from $M \times M$ we have $V^{1}\left(\widehat{\tau}_{1}, \widehat{\tau}_{2}\right) \geq V^{1}\left(\tau_{1}, \widehat{\tau}_{2}\right)$ and $V^{2}\left(\widehat{\tau}_{1}, \widehat{\tau}_{2}\right) \geq V^{2}\left(\widehat{\tau}_{1}, \tau_{2}\right)$.

Similarly as in the case of zero-sum stopping games the game $\mathcal{G}$ may not have a Nash equilibrium strategy unless sequences of rewards satisfy some regularity conditions. For instance, Ohtsubo (1987) proved existence of Nash equilibrium assuming that sequences $\left\{\left(X_{n}^{i}, Y_{n}^{i}, W_{n}^{i}\right), n \in N\right\}$ satisfy the integrability condition (1.2) and the inequalities $X_{n}^{i} \geq$ $W_{n}^{i} \geq Y_{n}^{i}$, a.s., for $i=1,2, n \in N$. Other types of constraints on reward sequences assuring existence of Nash equilibrium in the class of nonrandomized stopping times were considered in Ohstubo (1991), Ferenstein (1992, 1993), Bobecka and Ferenstein (2001). Finite horizon stopping games were investigated in a series of papers, often in the context of best choice problems. A broad survey of stopping games is given in Nowak and Szajowski (1999), Neumann, Ramsey and Szajowski (2002). 
The two-person nonzero-sum stopping game with priorities analyzed in Ferenstein (1993) has players' rewards

$$
\begin{aligned}
& R^{1}\left(\tau_{1}, \tau_{2}\right)=X_{\tau_{1}} I_{\left\{\tau_{1} \leq \tau_{2}\right\}}+Y_{\tau_{2}} I_{\left\{\tau_{2}<\tau_{1}\right\}}+\limsup _{n \rightarrow \infty} W_{n}^{1} I_{\left\{\tau_{1}=\tau_{2}=\infty\right\}}, \\
& R^{2}\left(\tau_{1}, \tau_{2}\right)=Y_{\tau_{1}} I_{\left\{\tau_{1} \leq \tau_{2}\right\}}+X_{\tau_{2}} I_{\left\{\tau_{2}<\tau_{1}\right\}}+\limsup _{n \rightarrow \infty} W_{n}^{2} I_{\left\{\tau_{1}=\tau_{2}=\infty\right\}},
\end{aligned}
$$

where $\left(\tau_{1}, \tau_{2}\right) \in M \times M, W_{n}^{1}=\max \left\{X_{n}, Y_{n}\right\}, W_{n}^{2}=\min \left\{X_{n}, Y_{n}\right\}, n \in N$.

Let us note that the two stage stopping games with priorities and reward sequences $\left\{X_{n}^{1}, n \in N\right\},\left\{X_{n}^{2}, n \in N\right\}$, described at the beginning, may be reduced to the above game if we put $X_{n}^{1}=X_{n}$, and $Y_{n}=\operatorname{ess} \sup \left\{E\left(X_{\tau}^{2} \mid \mathcal{F}_{n}\right): \tau \in M, \tau \geq n\right.$, a.s., $\left.\tau \in M\right\}$. Here, $X_{n}$ is interpreted as the reward for the player making selection first, with the constraint: if they both want to stop then Player 1 has the priority in getting the reward $X_{n}$, his opponent gets the reward $Y_{n}$.

Two-person nonzero-sum stopping games with rewards (1.5) and randomized stopping times were analyzed in Ferenstein (2005). Special type of $m$-person randomized stopping games with deterministic rewards, called quitting games, was considered by Solan and Vieille (2001). They obtained existence of Nash $\epsilon$-equilibrium strategies under some inequality constraints on rewards approaching infinity.

2. Model of the game. Let $(\Omega, \mathcal{F}, P)$ be an underlying probability space on which all considered random variables are defined and $\left\{\mathcal{F}_{n}, n \in N\right\}$ an increasing sequence of sub$\sigma$-fields of $\mathcal{F}, N=\{1,2, \ldots\} . \mathcal{F}_{n}$ is a $\sigma$-field of events observed by the players till time $n$, $n \in N$, inclusive. Let $M$ denote the set of stopping times $\tau$ with respect to $\left\{\mathcal{F}_{n}, n \in \bar{N}\right\}$, $\bar{N}=N \cup\{\infty\}, \mathcal{F}_{\infty}=\sigma\left(\left\{\mathcal{F}_{n}, n \in N\right\}\right.$, that is $\tau: \Omega \rightarrow \bar{N}$ and $\{\omega \in \Omega: \tau(\omega)=n\} \in \mathcal{F}_{n}$, for any $n \in \bar{N}$. Let $\left\{X_{n}^{j}\right\}, j=k, k-1, \ldots, 1$, be sequences of univariate random variables, $\left\{\mathcal{F}_{n}, n \in N\right\}$ adapted and satisfying the following condition

(A) $E\left(\sup _{n \in N} \max \left\{\left|X_{n}^{j}\right|, j=1,2, \ldots, k\right\}\right)<\infty$,

which are observed sequentially by $k$ ordered players. Below, we describe admissible game strategies. Each of the players is allowed to select only one reward from the sequences of rewards under the following constraints.

Let $j=k$ denote stage 1 of the game with $k$ ordered players. At this stage the players observe sequentially rewards $X_{n}^{k}, n=1,2, \ldots$, until the first time $n_{1}$, say, when at least one of them makes a decision to quit the game and get the current reward $X_{n}^{k}$ in which case the reward is assigned to the player with the highest priority (lowest rank) $i_{1}$, say, among those who have decided to get it. The remaining $k-1$ players take part at stage 2 of the game and observe sequentially, step by step, elements of the sequence of rewards $\left\{X_{n}^{k-1}\right\}_{\dot{n}>n_{1}}$. At the first time, $n_{2}$, say, when at least one of the players decides to quit the game stage 2 is terminated and the player with the lowest rank $i_{2}$, say, gets the reward $X_{n_{2}}^{k-1}$ and quits the game. In general, at any stage $l$ of the game, there are $k-l+1$ players who have not quit the game and have not obtained rewards at previous stages. They sequentially observe rewards $\left\{X_{n}^{k-l+1}\right\}_{\dot{n}>n_{l-1}}$, where $n_{l-1}$ is the time of terminating the $l-1$-th stage of the game. Stage $l$ terminates at time $n_{l}$, say, when Player $i_{l}$, say, gets the reward $X_{n_{l}}^{k-l+1}$, assuming the priorities in the selection scheme, 
that is the players with lower ranks have rejected it. Thus, one may introduce the set $H$ of possible game histories so that $h \in H$ if and only if $h=\left(n_{1}, i_{1}, n_{2}, i_{2}, \ldots, n_{k}, i_{k}\right)$, where $1 \leq n_{1}<n_{2}<\cdots<n_{k}<\infty, i_{j} \in\{1,2, \ldots, k\}, i_{r} \neq i_{j}$ for $r \neq j$.

Now, we may define the set $S$ of the players' strategies. Let $D$ be the set of sequences $\left\{p_{n}\right\}_{n \in N}$ of 0 -1 valued random variables, $\left\{\mathcal{F}_{n}\right\}_{n \in N}$ adapted. For $i=1,2, \ldots, k, s^{i} \in$ $S$ is a strategy of Player $i$ if and only if $s^{i}: H \rightarrow D \times D \times \cdots \times D$, and $s^{i}(h)=$ $\left(s_{1}^{i}\left(h_{0}\right), s_{2}^{i}\left(h_{1}\right), \ldots, s_{k}^{i}\left(h_{k-1}\right)\right), h \in H, h_{l}=\left(n_{1}, i_{1}, \ldots, n_{l}, i_{l}\right), l=1,2, \ldots, k-1, h_{0}=$ $(0,0)$, where $s_{l}^{i}\left(h_{l-1}\right)=\left\{p_{n, l}^{i}\left(h_{l-1}\right)\right\} \in D$ is the strategy at stage $l$ such that $p_{n, l}^{i}\left(h_{l-1}\right)=$ 0 if $i \in h_{l-1}$ and $p_{n, l}^{i}\left(h_{l-1}\right)=0$ if $i \notin h_{l-1}, n \leq n_{l-1}$. The $k$-tuple of players' strategies $s=\left(s^{1}, s^{2}, \ldots, s^{k}\right) \in S \times S \times \cdots \times S$ is called the game strategy. Below, we describe the mechanism by which a strategy $s$ determines an evolution of the game, that is stopping times $t_{l}, l=1,2, \ldots, k$, of terminating each stage $l$ of the game and players $L_{l}$ who obtain rewards at stage $l$.

Let $s \in S \times S \times \cdots \times S$. For $l=1,2, \ldots, k$, define recursively the stopping times $\tau_{l}^{i}$, $t_{l}, i=1,2, \ldots, k$, and random players' ranks (indices) $L_{l}$, as follows:

$\tau_{1}^{i}=\inf \left\{n \geq 1: p_{n, 1}^{i}\left(H_{0}\right)=1\right\}, t_{1}=\inf \left\{\tau_{1}^{i}, i=1,2, \ldots, k\right\}, L_{1}=\min \{i \in$ $\left.\{1,2, \ldots, k\}: t_{1}=\tau_{1}^{i}\right\}, H_{0}=h_{0}$.

Suppose that, for given $1 \leq l \leq k-1$, random variables $\tau_{l}^{i}, t_{l}, L_{l}$ have been defined and denote $H_{l}=\left(t_{1}, L_{1}, \ldots, t_{l}, L_{l}\right)$. Then, define for $i=1,2, \ldots, k$,

$\tau_{l+1}^{i}=\inf \left\{n>t_{l}: p_{n . l+1}^{i}\left(H_{l}\right)=1\right\}$ if $i \neq L_{1}, \ldots, L_{l}$, and $\tau_{l+1}^{i}=\infty$, otherwise,

$t_{l+1}=\inf \left\{\tau_{l+1}^{i}, i \in\{1,2, \ldots, k\}, i \neq L_{1}, \ldots, L_{l}\right\}, L_{l+1}=\min \{i \in\{1,2, \ldots, k\}: i \neq$ $\left.L_{1}, \ldots, L_{l}, t_{l+1}=\tau_{l+1}^{i}\right\}$.

Now, the reward $R^{i}(s)$ for Player $i, i=1,2, \ldots, k$, and his mean reward $V^{i}(s)$ under the game strategy $s \in S \times S \times \cdots \times S$ are defined as follows

$$
\begin{gathered}
R^{i}(s)=\sum_{l=1}^{k} I_{\left\{L_{l}=i\right\}} X_{t_{l}}^{k-l+1}, \\
V^{i}(s)=E\left[R^{i}(s)\right]
\end{gathered}
$$

where $I_{A}$ is the indicator function of the event $A$.

The considered game may be written in a normal way as $\mathcal{G}=\left(S \times S \times \cdots \times S, V^{1}, V^{2}\right.$, $\left.\ldots, V^{k}\right)$. The aim of each of the players is to make his mean reward as large as possible. Hence equilibrium strategies are of interest. For $s=\left(s_{1}, s_{2}, \ldots, s_{k}\right) \in S \times S \times \cdots \times S$, $\widehat{s}_{i} \in S, i=1,2, \ldots, k$, denote $\left(s^{-i}, \widehat{s}_{i}\right)=\left(s_{1}, \ldots, s_{i-1}, \widehat{s}_{i}, \ldots, s_{k}\right)$. Let us recall that a game strategy $\widehat{s} \in S \times S \times \cdots \times S$ is a Nash equilibrium strategy if for any strategy $s \in S \times S \times \cdots \times S$ and any Player $i, i=1,2, \ldots, k$, we have

$$
V^{i}(\widehat{s}) \geq V^{i}\left(\widehat{s}^{-i}, s_{i}\right) \text {. }
$$

3. Nash equilibria. In this section we will describe a way to construct Nash equilibria of the game $\mathcal{G}$, using the dynamic programming approach and general results on optimal stopping, Chow, Robbins and Siegmund (1971), and results on two-person stopping games with priorities obtained in Enns and Ferenstein (1987), Ferenstein (1993).

Consider the final stage $k$, when there is one player left, player $L_{k}$, and he observes step by step rewards $X_{t_{k-1}+j}^{1}, j=1,2, \ldots$ Let us recall that $X_{n}^{l}$ stands for the reward 
at $n$ at stage $k-l+1$, that is there are $l$ players left. We will describe heuristically the way to obtain a reasonable Nash equilibrium strategy and sequences of optimal players' rewards. First, we will construct $k$ sequences of Bellman equations.

Consider the final stage of the game. There is one player left, and he starts to observe sequentially rewards just after the time of stopping at the previous stage $t_{k-1}=n_{k-1}$. Thus, his policy is to find an optimal stopping time for the sequence $X_{n}^{1}, n=n_{k-1}+$ $1, n_{k-1}+2, \ldots$. Let the corresponding sequence of optimal conditional rewards be denoted by $\left\{\gamma_{n, 1}^{1}\right\}$. Hence,

$$
\gamma_{n, 1}^{1}=\operatorname{ess} \sup \left\{E\left(X_{\tau}^{1} \mid \mathcal{F}_{n}\right): \tau \in M, \tau \geq n, \text { a.s. }\right\},
$$

and, for any $l=1, \ldots, k$, an optimal time of final selection is

$$
\widehat{\tau}_{k}^{1}=\inf \left\{n>t_{k-1}: X_{n}^{1}=E\left(\gamma_{n+1,1}^{1} \mid \mathcal{F}_{n}\right)\right\} \text {. }
$$

Now, suppose that there are 2 players left and they begin to observe the reward sequence $X_{n}^{2}, n>t_{k-2}=n_{k-2}$, say. Then, the player who decides to quit the game while his opponent decides to continue it gets a reward $X_{n}^{2}$ or if both players are going to quit the game then the player with the higher priority gets the reward. The remaining player begins to observe the sequence $\left\{X_{n+j}^{1}\right\}_{j \in N}$ of rewards of the final stage of the game. Thus, his optimal conditional reward for continuing the game after time $n$ given $\mathcal{F}_{n}$ is $E\left(\gamma_{n+1,1}^{1} \mid \mathcal{F}_{n}\right)$. For a given policy up to stage $k-2$ and such that $t_{k-1} \geqslant n$, let us denote by $\gamma_{n, 2}^{j}, j=1,2$, the optimal conditional reward for the player with relative order $j$, given $\mathcal{F}_{n}$. Then, because of priorities and our assumption that at the final stage the player acts according to an optimal stopping rule, it is easy to suspect that the sequences $\left\{\gamma_{n, 2}^{j}\right\}$ satisfy the following Bellman equations under an optimal strategy:

$$
\begin{aligned}
& \gamma_{n, 2}^{1}=X_{n}^{2} \text { if } X_{n}^{2} \geq \max \left\{E\left(\gamma_{n+1,1}^{1} \mid \mathcal{F}_{n}\right), E\left(\gamma_{n+1,2}^{2} \mid \mathcal{F}_{n}\right)\right\}, \\
& \gamma_{n, 2}^{1}=E\left(\gamma_{n+1,1}^{1} \mid \mathcal{F}_{n}\right) \text { if } E\left(\gamma_{n+1,2}^{2} \mid \mathcal{F}_{n}\right) \leq X_{n}^{2}<E\left(\gamma_{n+1,1}^{1} \mid \mathcal{F}_{n}\right), \\
& \gamma_{n, 2}^{1}=E\left(\gamma_{n+1,2}^{1} \mid \mathcal{F}_{n}\right) \text { if } E\left(\gamma_{n+1,2}^{2} \mid \mathcal{F}_{n}\right)>X_{n}^{2}
\end{aligned}
$$

and

$$
\begin{aligned}
& \gamma_{n, 2}^{2}=X_{n}^{2} \text { if } E\left(\gamma_{n+1,2}^{2} \mid \mathcal{F}_{n}\right) \leq X_{n}^{2}<E\left(\gamma_{n+1,1}^{1} \mid \mathcal{F}_{n}\right), \\
& \gamma_{n, 2}^{2}=E\left(\gamma_{n+1,2}^{2} \mid \mathcal{F}_{n}\right) \text { if } E\left(\gamma_{n+1,2}^{2} \mid \mathcal{F}_{n}\right)>X_{n}^{2}, \\
& \gamma_{n, 2}^{2}=E\left(\gamma_{n+1,1}^{1} \mid \mathcal{F}_{n}\right) \text { if } X_{n}^{2} \geq \max \left\{E\left(\gamma_{n+1,1}^{1} \mid \mathcal{F}_{n}\right), E\left(\gamma_{n+1,2}^{2} \mid \mathcal{F}_{n}\right)\right\} .
\end{aligned}
$$

Proposition 1. There exist $\left\{\mathcal{F}_{n}, n \in N\right\}$ adapted sequences $\left\{\gamma_{n, 2}^{j}\right\}, j=1,2$, satisfying equations (3.2) and (3.3).

To solve these equations it is sufficient to prove the existence of a sequence $\left\{\gamma_{n, 2}^{2}\right\}$ satisfying the last three equalities (3.3) which is a direct consequence of Lemma 2 below proved in Ferenstein (1993), where we put $X_{n}=X_{n}^{2}, Y_{n}=E\left(\gamma_{n+1,1}^{1} \mid \mathcal{F}_{n}\right), G_{n}=$ $\min \left\{X_{n}, Y_{n}\right\}$.

Lemma 2. Suppose that $\left\{X_{n}\right\}_{n \in N},\left\{Y_{n}\right\}_{n \in N}$ are $\left\{\mathcal{F}_{n}, n \in N\right\}$ adapted sequences of univariate random variables and let $G_{n}=\min \left\{X_{n}, Y_{n}\right\}, n \in N$. Let $E\left(\sup _{n \in N} G_{n}^{+}\right)<\infty$ and $E\left(G_{n}^{-}\right)<\infty, n \in N$. Then, there exists an $\left\{\mathcal{F}_{n}\right\}_{n \in N}$ adapted sequence of random variables $\left\{\mu_{n}\right\}_{n \in N}$ satisfying the following conditions: 
(i) $G_{n} \leq \mu_{n} \leq \operatorname{ess} \sup \left\{E\left(G_{\tau} \mid \mathcal{F}_{n}\right): \tau \in M, \tau \geq n\right.$,a.s. $\}$.

(ii) $\mu_{n}= \begin{cases}G_{n} & \text { if } X_{n}>E\left(\mu_{n+1} \mid \mathcal{F}_{n}\right), \\ E\left(\mu_{n+1} \mid \mathcal{F}_{n}\right), & \text { otherwise. }\end{cases}$

Proposition 3 below is a direct generalization of Lemma 2 suitable for our $k$-person stopping game.

Proposition 3. Suppose that $\left\{X_{n}^{l}\right\}_{n \in N}, l=1,2, \ldots, k$, are $\left\{\mathcal{F}_{n}, n \in N\right\}$ adapted sequences of univariate random variables such that $E\left(\max \left\{\sup _{n \in N} X_{n}^{1+}, \ldots, \sup _{n \in N} X_{n}^{k+}\right)\right\}$ $<\infty$ and $E\left(X_{n}^{l-}\right)<\infty, n \in N, l=1,2, \ldots, k$. Then, there exist $\left\{\mathcal{F}_{n}\right\}_{n \in N}$ adapted sequences of random variables $\left\{\gamma_{n, l}^{j}\right\}_{n \in N}, j=1, \ldots, l, l=1,2, \ldots, k$, satisfying the following conditions:

(C1) $\gamma_{n, 1}^{1}=\operatorname{ess} \sup \left\{E\left(X_{\tau}^{1} \mid \mathcal{F}_{n}\right): \tau \in M, \tau \geq n\right.$, a.s. $\}$.

(C2) $\gamma_{n, j}^{j}= \begin{cases}G_{n}^{j} & \text { if } X_{n}^{j} \geq E\left(\gamma_{n+1, j}^{j} \mid \mathcal{F}_{n}\right), \\ E\left(\gamma_{n+1, j}^{j} \mid \mathcal{F}_{n}\right) & \text { otherwise, a.s., }\end{cases}$

where $G_{n}^{j}=\min \left\{X_{n}^{j}, E\left(\gamma_{n+1, j-1}^{j-1} \mid \mathcal{F}_{n}\right)\right\}, j=2, \ldots, k$.

(C3) $G_{n}^{j} \leq \gamma_{n, j}^{j} \leq \operatorname{ess} \sup \left\{E\left(G_{\tau}^{j} \mid \mathcal{F}_{n}\right): \tau \in M, \tau \geq n\right.$,a.s. $\}$, a.s.

Let us define recursively $k$ multivariate random sequences, for $j=1,2, \ldots, k,\left\{\gamma_{n, j}^{j}\right.$, $\left.\gamma_{n, j}^{j-1}, \gamma_{n, j}^{j-2}, \ldots, \gamma_{n, j}^{1}\right\}_{n \in N}$, as follows. For $j=1, \ldots, k,\left\{\gamma_{n, j}^{j}\right\}$ is a sequence satisfying Conditions (C1)-(C3) of Proposition 3. For the fixed $j>1$, suppose that the sequence $\left\{\gamma_{n, j-1}^{l}, l=1, \ldots, j-1\right\}_{n \in N}$ has been defined. Define
(C4) $\gamma_{n, j}^{1}=\left\{\begin{array}{l}X_{n}^{j} \\ E\left(\gamma_{n+1, j-1}^{1} \mid \mathcal{F}_{n}\right) \\ E\left(\gamma_{n+1, j}^{1} \mid \mathcal{F}_{n}\right)\end{array}\right.$
if $X_{n}^{j} \geq \max \left\{E\left(\gamma_{n+1, j}^{j} \mid \mathcal{F}_{n}\right), E\left(\gamma_{n+1, j-1}^{1} \mid \mathcal{F}_{n}\right)\right\}$,
if $\left.E\left(\gamma_{n+1, j}^{j} \mid \mathcal{F}_{n}\right) \leq X_{n}^{j}<E\left(\gamma_{n+1, j-1}^{1} \mid \mathcal{F}_{n}\right)\right\}$,
otherwise, a.s.

For $l=2, \ldots, j-1$, let $\gamma_{n, j}^{l}$ be defined as follows

$$
(\mathrm{C} 5) \gamma_{n, j}^{l}= \begin{cases}X_{n}^{j} & \text { if } X_{n}^{j} \geq \max \left\{E\left(\gamma_{n+1, j}^{j} \mid \mathcal{F}_{n}\right), E\left(\gamma_{n+1, j-1}^{l} \mid \mathcal{F}_{n}\right)\right\}, \\ & \text { and } X_{n}^{j}<E\left(\gamma_{n+1, j-1}^{l-1} \mid \mathcal{F}_{n}\right), \\ E\left(\gamma_{n+1, j-1}^{l-1} \mid \mathcal{F}_{n}\right) & \text { if } X_{n}^{j} \geq \max \left\{E\left(\gamma_{n+1, j}^{j} \mid \mathcal{F}_{n}\right), E\left(\gamma_{n+1, j-1}^{l-1} \mid \mathcal{F}_{n}\right)\right\} \\ E\left(\gamma_{n+1, j-1}^{l} \mid \mathcal{F}_{n}\right) & \text { if } E\left(\gamma_{n+1, j-1}^{l-1} \mid \mathcal{F}_{n}\right)>X_{n}^{j} \geq E\left(\gamma_{n+1, j}^{j} \mid \mathcal{F}_{n}\right), \\ =E\left(\gamma_{n+1, j}^{l} \mid \mathcal{F}_{n}\right) & \text { otherwise, a.s. }\end{cases}
$$

Moreover, let us introduce the events:

$$
\begin{aligned}
& A_{n, j}^{j}=\left\{X_{n}^{j} \geq E\left(\gamma_{n+1, j}^{j} \mid \mathcal{F}_{n}\right)\right\}, j=1,2, \ldots, k, \\
& A_{n, j}^{1}=\left\{X_{n}^{j} \geq E\left(\gamma_{n+1, j-1}^{1} \mid \mathcal{F}_{n}\right)\right\} \cap A_{n, j}^{j}, \\
& A_{n, j}^{l}=\left\{E\left(\gamma_{n+1, j-1}^{l} \mid \mathcal{F}_{n}\right) \leq X_{n}^{j}<E\left(\gamma_{n+1, j-1}^{l-1} \mid \mathcal{F}_{n}\right)\right\} \cap A_{n, j}^{j}, l=2, \ldots, j-1, \\
& A_{n, j}^{0}=\Omega-A_{n, j}^{j} .
\end{aligned}
$$

We will show that under an equilibrium strategy described in some special way by the above sequences of random variables the event $A_{n, j}^{j}$ consists of outcomes for which at $n$ stage $k-j+1$ is terminated. The remaining events determine players who quits the 
game: $A_{n, j}^{l}$ is the set of outcomes for which the player with the relative order $l$ quit the game at $n$ at stage $k-j+1$, that is there are still $j$ players in the game.

Given the multivariate sequences $\left\{\gamma_{n, j}^{j}, \gamma_{n, j}^{j-1}, \gamma_{n, j}^{j-2}, \ldots, \gamma_{n, j}^{1}\right\}_{n \in N}, j=1,2, \ldots, k$, let us define for $j=1, \ldots, k, l=1, \ldots, j$, and the history $h$, random sequences $\left\{p_{n, j}^{l}\left(h_{j-1}\right)\right\}$ by

(D) $p_{n, j}^{l}\left(h_{j-1}\right)(\omega)= \begin{cases}1 & \text { if } \omega \in A_{n, k-j+1}^{l} \\ 0 & \text { otherwise }\end{cases}$

Theorem 4. Assume that Condition (A) is satisfied, $\left\{\gamma_{n, j}^{j}, \gamma_{n, j}^{j-1}, \gamma_{n, j}^{j-2}, \ldots, \gamma_{n, j}^{1}\right\}_{n \in N}, j=$ $1,2, \ldots, k$, are $\left\{\mathcal{F}_{n}\right\}_{n \in N}$ adapted sequences of random variables satisfying Conditions (C1)-(C5). Let $\widehat{s}=\left(\widehat{s}^{1}, \widehat{s}^{2}, \ldots, \widehat{s}^{k}\right) \in S \times S \times \cdots \times S$ be the game strategy such that for any $h \in H, i, j=1,2, \ldots, k$,

$$
\widehat{s}_{j}^{i}\left(h_{j-1}\right)= \begin{cases}\left\{p_{n, j}^{r(i)}\left(h_{j-1}\right)\right\}, & \text { if } i \notin h_{j-1}, \\ \{0\} & \text { otherwise, }\end{cases}
$$

where $r(i)$ is the relative rank of Player $i$ among all Players $l \notin h_{j-1}$. Then, $\hat{s}$ is an equilibrium strategy and

$$
V^{i}(\widehat{s})=E\left(\gamma_{1, k}^{i}\right), i=1, \ldots, k .
$$

Proof. The proof is by a dynamic programming technique with respect to the game stage $l: l=k, k-1, \ldots, 1$, and for each stage $l$ given the history $h_{l-1}$ with respect to time $n$ $>n_{l-1}$, similarly as in optimal stopping time problems and using results obtained in Ferenstein (1993) for the case of two players. For instance, let $i<j, i, j \in\{1, \ldots, k\}$, be relative order of two players who have remained in the game at stage $k-1$. Then, following Theorems 1 and 2 in Ferenstein (1993), we easily get that the following stopping times are optimal Nash equilibrium stopping times:

$$
\begin{aligned}
& \widehat{\tau}_{k-1}^{1}=\inf \left\{n>t_{k-2}: X_{n}^{2} \geq \max \left\{E\left(\gamma_{n+1,2}^{2} \mid \mathcal{F}_{n}\right), E\left(\gamma_{n+1,1}^{1} \mid \mathcal{F}_{n}\right)\right\},\right. \\
& \widehat{\tau}_{k-1}^{2}=\inf \left\{n>t_{k-2}: E\left(\gamma_{n+1,1}^{1} \mid \mathcal{F}_{n}\right)>X_{n}^{2} \geq E\left(\gamma_{n+1,2}^{2} \mid \mathcal{F}_{n}\right) .\right.
\end{aligned}
$$

4. Special classes. Suppose that a reward at any stage of the game and any time is some function of the currently observed state of the Markov chain $\left\{\xi_{n}: n \in N\right\}$. Hence, $\left\{X_{n}^{i}\right\}_{n \in N}=\left\{g\left(\xi_{n}\right)\right\}_{n \in N}, i=1,2, \ldots, k$. Suppose that there is an equilibrium strategy. We will derive a "reasonable" Nash equilibrium strategy heuristically, first. Let $\gamma_{n, l}^{j}$, $j=1, \ldots, l, l \in\{1, \ldots, k\}$, be the optimal conditional reward given $\mathcal{F}_{n}$ for the player with relative rank $j$ among all $l$ players still be active in the game at stage $k-l+1$ at time $n$. At stage $k$, the optimal remaining player's strategy is just to stop as in the classical optimal stopping time problem. Hence, optimal stopping time of the last selection is $t_{k}=\tau_{k}=$ $\inf \left\{n \geq t_{k-1}+1: g\left(\xi_{n}\right)=E\left(\gamma_{n+1,1}^{1} \mid \mathcal{F}_{n}\right)\right.$, where $\gamma_{n, 1}^{1}=\operatorname{ess} \sup \left\{E\left(g\left(\xi_{\tau}\right) \mid \mathcal{F}_{n}\right), \tau \geq n\right\}$. Suppose that there are 2 players left at stage $k-1$. Then, assuming $t_{k-2}=n_{k-2}$, we have a two-person nonzero-sum game with priorities starting at $n_{k-2}+1$ with the rewards given by (1.6) and (1.7), where $X_{n}=g\left(\xi_{n}\right), Y_{n}=E\left(\gamma_{n+1,1}^{1} \mid \mathcal{F}_{n}\right)$. The way of constructing sequences $\gamma_{n+1,2}^{1}, \gamma_{n+1,2}^{2}$ is described in Ferenstein (1993) as well as stopping policy which is fully determined by $t_{k-1}$ and the relative rank of the player getting the 
reward $g\left(\xi_{t_{k-1}}\right)$, denoted by $r_{2}, r_{2} \in\{1,2\}$. Let us consider now the case when there are 3 players left at stage $k-2$. Suppose $t_{k-3}=n_{k-3}$ and until time $n>n_{k-3}$ nobody has selected the reward at this stage. Then, the following cases (a) - (d) are possible, noting that for "reasonable" strategies we have

$$
\begin{gathered}
E\left(\gamma_{n+1,2}^{1} \mid \mathcal{F}_{n}\right) \geq E\left(\gamma_{n+1,3}^{1} \mid \mathcal{F}_{n}\right), \text { a.s. } \\
\gamma_{n+1,3}^{1} \geqslant \gamma_{n+1,3}^{2} \geqslant \gamma_{n+1,3}^{3}, \text { a.s. }
\end{gathered}
$$

(a) $g\left(\xi_{n}\right) \geq E\left(\gamma_{n+1,2}^{1} \mid \mathcal{F}_{n}\right) \geq E\left(\gamma_{n+1,3}^{1} \mid \mathcal{F}_{n}\right)$, then $t_{k-2}=\tau_{k-2}^{1}=n$ and $r_{3}=1$, where $r_{3}$ denote the relative rank of the player stopping.

(b) $E\left(\gamma_{n+1,3}^{3} \mid \mathcal{F}_{n}\right) \leq E\left(\gamma_{n+1,2}^{2} \mid \mathcal{F}_{n}\right) \leq g\left(\xi_{n}\right)<E\left(\gamma_{n+1,2}^{1} \mid \mathcal{F}_{n}\right)$, then $t_{k-2}=\tau_{k-2}^{2}=n$ and $r_{3}=2$.

(c) $E\left(\gamma_{n+1,3}^{3} \mid \mathcal{F}_{n}\right) \leq g\left(\xi_{n}\right)<E\left(\gamma_{n+1,2}^{2} \mid \mathcal{F}_{n}\right)$, then $r_{3}=3, t_{k-2}=\tau_{k-2}^{3}=n$.

(d) $E\left(\gamma_{n+1,3}^{3} \mid \mathcal{F}_{n}\right)>g\left(\xi_{n}\right)$, then nobody stops at time $n$, that is $\tau_{k-2}^{i}>n, i=1,2,3$.

The following events are Player's 1,2,3 stopping events:

$$
\begin{aligned}
& A_{n, 3}^{1}=\left\{g\left(\xi_{n}\right) \geq E\left(\gamma_{n+1,2}^{1} \mid \mathcal{F}_{n}\right)\right\}, \\
& A_{n, 3}^{2}=\left\{E\left(\gamma_{n+1,3}^{2} \mid \mathcal{F}_{n}\right) \leq g\left(\xi_{n}\right)<E\left(\gamma_{n+1,2}^{1} \mid \mathcal{F}_{n}\right)\right\}, \\
& A_{n, 3}^{3}=\left\{E\left(\gamma_{n+1,3}^{3} \mid \mathcal{F}_{n}\right) \leq g\left(\xi_{n}\right)<E\left(\gamma_{n+1,3}^{2} \mid \mathcal{F}_{n}\right)\right\}, \text { and } \\
& A_{n, 3}^{0}=\left\{g\left(\xi_{n}\right)<E\left(\gamma_{n+1,3}^{3} \mid \mathcal{F}_{n}\right)\right\} \text { denotes the event "not stopping at time } n " .
\end{aligned}
$$

Then, optimal rewards at time $n$ at stage $k-2$ are

$$
\begin{aligned}
\gamma_{n, 3}^{1}= & g\left(\xi_{n}\right) I\left(A_{n, 3}^{1}\right)+E\left(\gamma_{n+1,2}^{1} \mid \mathcal{F}_{n}\right) I\left(A_{n, 3}^{2} \cup A_{n, 3}^{3}\right)+E\left(\gamma_{n+1,3}^{1} \mid \mathcal{F}_{n}\right) I\left(A_{n, 3}^{0}\right), \\
\gamma_{n, 3}^{2}= & E\left(\gamma_{n+1,2}^{1} \mid \mathcal{F}_{n}\right) I\left(A_{n, 3}^{1}\right)+E\left(\gamma_{n+1,2}^{2} \mid \mathcal{F}\right) I\left(A_{n, 3}^{3}\right)+g\left(\xi_{n}\right) I\left(A_{n, 3}^{2}\right) \\
& +E\left(\gamma_{n+1,3}^{2} \mid \mathcal{F}_{n}\right) I\left(A_{n, 3}^{0}\right), \\
\gamma_{n, 3}^{3}= & E\left(\gamma_{n+1,2}^{2} \mid \mathcal{F}_{n}\right) I\left(A_{n, 3}^{1} \cup A_{n, 3}^{2}\right)+g\left(\xi_{n}\right) I\left(A_{n, 3}^{3}\right)+E\left(\gamma_{n+1,3}^{3} \mid \mathcal{F}_{n}\right) I\left(A_{n, 3}^{0}\right) .
\end{aligned}
$$

Similar motivation as above leads us to obtain sequences of optimal conditional rewards at any $(k-l)$-th stage of the game with priority when there are $l$ players who have not chosen rewards before. These are $\left\{\gamma_{n, l}^{j}, n \geq k-l+1\right\}, j=1, \ldots, l$, where $\gamma_{n, l}^{j}$ denotes the optimal conditional reward of the player with relative rank $j$ among $l$ players who are still at the game. Let $A_{n, l}^{j}$ be a stopping set of the player with relative rank $j$ at $n$ and let $A_{n, l}^{0}$ denotes the event "not to stop" at time $n$. Then,

$$
\begin{aligned}
\gamma_{n, l}^{j}= & g\left(\xi_{n}\right) I\left(A_{n, l}^{j}\right)+E\left(\gamma_{n+1, l-1}^{j-1} \mid \mathcal{F}_{n}\right) I\left(\bigcup_{i<j} A_{n, l}^{i}\right) \\
& +E\left(\gamma_{n+1, l-1}^{j} \mid \mathcal{F}_{n}\right) I\left(\bigcup_{i>j} A_{n, l}^{i}\right)+E\left(\gamma_{n+1, l}^{j} \mid \mathcal{F}_{n}\right) I\left(A_{n, l}^{0}\right),
\end{aligned}
$$

where $j=1, \ldots, l ; l=3, \ldots, k ; \gamma_{n+1, l-1}^{0}=\gamma_{n+1, l-1}^{1}$, and stopping sets at $n$ are

$$
\begin{aligned}
& A_{n, l}^{1}=\left\{g\left(\xi_{n}\right) \geq E\left(\gamma_{n+1, l-1}^{1} \mid \mathcal{F}_{n}\right)\right\}, \\
& A_{n, l}^{j}=\left\{E\left(\gamma_{n+1, l}^{j} \mid \mathcal{F}_{n}\right) \leq g\left(\xi_{n}\right)<E\left(\gamma_{n+1, l-1}^{j-1} \mid \mathcal{F}_{n}\right)\right\}, j=2, \ldots, l-1, \\
& A_{n, l}^{l}=\left\{E\left(\gamma_{n+1, l}^{l} \mid \mathcal{F}_{n}\right) \leq g\left(\xi_{n}\right)<E\left(\gamma_{n+1, l-1}^{l-1} \mid \mathcal{F}_{n}\right)\right\},
\end{aligned}
$$

and

$$
A_{n, l}^{0}=\left\{g\left(\xi_{n}\right)<E\left(\gamma_{n+1, l}^{l} \mid \mathcal{F}_{n}\right)\right\}
$$


is the set of rejecting rewards at $n$, that is all remaining players decide not to quit the game at $n$ and observe rewards at time $n+1$.

\section{References}

K. Bobecka and E. Z. Ferenstein (2001), On nonzero-sum stopping game related to discrete risk process, Control and Cybernetics 30, 339-354.

Y. S. Chow, H. Robbins and D. Siegmund (1971), Great Expectations: The Theory of Optimal Stopping, Houghton Mifflin Co., Boston.

E. B. Dynkin (1969), Game variant of a problem on optimal stopping, Soviet Math. Dokl. 10, 270-274.

E. G. Enns and E. Z. Ferenstein (1987), On a multi-person time-sequential game with priorities, Sequential Analysis 6, 239-256.

E. Z. Ferenstein (1992), Two-person non-zero-sum game with priorities, in: T. S. Ferguson and S. M. Samuels (eds.), Contemporary Mathematics 125, 119-133.

E. Z. Ferenstein (1993), A variation of the Dynkin's stopping game, Mathematica Japonica 38, 371-379.

E. Z. Ferenstein (2005), On randomized stopping games, in: A. S. Nowak and K. Szajowski (eds.), Annals of the International Society of Dynamic Games 7, Birkhäuser, 223-233.

J. Nash (1951), Non-cooperative games, Ann. Math. 54, 286-295.

J. Neveu (1975), Discrete Parameter Martingales, North-Holland, Amsterdam.

P. Neumann, D. Ramsey and K. Szajowski (2002), Randomized stopping times in Dynkin games, ZAMM Z. Angew. Math. Mech. 82, 811-819.

A. S. Nowak and K. Szajowski (1999), Nonzero-sum stochastic games, Annals of the International Society of Dynamic Games, 4, 297-343.

Y. Ohtsubo (1987), A nonzero-sum extension of Dynkin's stopping problem, Mathematics of Operations Research 12, 277-296.

Y. Ohtsubo (1991), On a discrete-time non-zero-sum Dynkin problem with monotonicity, J. Appl. Probab. 28, 466-472.

D. Rosenberg, E. Solan and N. Vieille (2000), Stopping games with randomized strategies, Technical Report 50, Laboratoire D'Econometrie Ecole Polytechnique, Paris.

E. Solan and N. Vieille (2000), Quitting games, Math. Oper. Res. 26, 265-285.

M. Yasuda (1985), On randomized strategy in Neveu's stopping problem, Stochastic Processes and their Applications 21, 159-166. 